\title{
Succinate dehydrogenase: the complex roles of a simple enzyme
}

Shaobai Huang and A. Harvey Millar

ARC Centre of Excellence in Plant Energy Biology and Centre for Comparative Analysis of Biomolecular Networks (CABiN), The University of Western Australia, Western Australia, Australia

\section{Highlights}

- The composition of plant SDH complex is different to those in other eukaryotes

- SDH mutation in plants changes organic acids, respiration and ROS generation

- SDH mutation alters photosynthesis, stomata, root elongation and fungal defence

- Tissue-specific phenotypes, ROS and succinate increases are key research topics 


\title{
Succinate dehydrogenase: the complex roles of a simple enzyme
}

Shaobai Huang and A. Harvey Millar

ARC Centre of Excellence in Plant Energy Biology and Centre for Comparative Analysis of Biomolecular Networks (CABiN), The University of Western Australia, Western Australia, Australia

harvey.millar@uwa.edu.au, shaobai.huang@uwa.edu.au

Corresponding author: A. Harvey Millar, +61 864887245 (phone), +61 864884401 (fax), harvey.millar @uwa.edu.au, Bayliss Building M316, The University of Western Australia, 35 Stirling Highway, Crawley WA 6009, Western Australia, Australia.

\begin{abstract}
Succinate dehydrogenase (SDH) oxidises succinate to fumarate as a component of the tricarboxylic acid cycle and ubiquinone to ubiquinol in the mitochondrial electron transport chain. Studies of SDH mutants have revealed far-reaching effects of altering succinate oxidation in plant cells. The plant SDH complex composition, structure and assembly are all beginning to be understood but the implications of the divergence across eukaryotes is still unclear. We propose an integration of the reported physiological roles of SDH in plants which influence photosynthesis, the function of stomata, root elongation and fungal defence. Future SDH research needed in plants should involve tissue-specific studies of mutants, analysis of the pathways induced by succinate-dependent reactive oxygen species generation and assessment of the impact of succinate accumulation on metabolism.
\end{abstract}




\section{Introduction}

Succinate dehydrogenase (SDH EC 1.3.99.1 and complex II, EC 1.3.5.1) has a central role in mitochondrial metabolism as the only enzyme that is a component of both the TCA cycle and the electron transport chain. SDH catalyzes the oxidation of succinate to fumarate in the mitochondria matrix and transfers electrons to ubiquinone without pumping protons across the mitochondrial inner membrane. $\mathrm{SDH}$ is allosterically regulated by the binding of oxaloacetate and is activated by ATP in the process that does not involve phosphorylation ([1] and refs therein). Distinct diseases have been shown to be associated with mutations in different subunits of SDH in humans [2]. Recently, the reasons for these phenotypic differences in human SDH mutations are starting to be revealed by in-depth studies of the consequences of interrupting succinate oxidation at different points in the catalytic operation of SDH [3]. In plants, mutations effecting different SDH subunits differentially affect roots, leaves and plant responses to the environment $\left[4^{*}, 5^{*}, 6^{*}\right.$, $7^{*}$ ]. In this review we attempt to integrate recent advances into the composition, assembly and physiological roles played by SDH in plants into a broader framework of the role of SDH in eukaryotes, and discuss key directions for exploring the roles of $\mathrm{SDH}$ in plants.

\section{Composition and assembly of SDH in plants}

The apparent diversity of SDH complex size and assembly among different organisms may underlie differences in the physiological function of SDH in these species. SDH in eukaryotes is classically comprised of four subunits (Figure 1), a flavoprotein (SDH1) that contains a bound FAD cofactor, an iron-sulfur ( $\mathrm{Fe}-\mathrm{S}$ ) protein $(\mathrm{SDH} 2)$ that contains three Fe-S clusters, and two small integral membrane proteins (SDH3 and SDH4) that bind heme to form the $b$ - type cytochrome $[8,9]$. The amino acid sequences of SDH1 and SDH2 share $80 \%$ identity across eukaryotes in key regions needed for succinate, FAD and Fe-S binding, showing the tight structure/function relationship in succinate oxidation. However, somewhat surprisingly, the sequences of $\mathrm{SDH} 3$ and $\mathrm{SDH} 4$ subunits and the ubiquinone binding regions have greatly diverged in plants, fungi and mammals (Figure 2), perhaps in part due to a complex genetic history of their switching from being encoded in nuclear and mitochondrial genes [10,11]. 
Despite much research on assembly of other complexes of the mitochondrial respiratory chain, very little was known until recently about the process of assembly of SDH. Two assembly factors for SDH, SDHAF1 and SDHAF2 (Figure 1), were recently reported in mammals as the cause of different human diseases [12,13]. SDHAF1 and SDHAF2 are proposed to function to insert Fe-S and FAD in SDH2 and SDH1, respectively $[12,13]$. An ortholog of human SDHAF2 was recently identified as an unknown function mitochondrial protein in the model plant Arabidopsis $\left[5^{\circ}, 14\right]$. This plant SDHAF2 has low amino acid sequence similarity to human and yeast SDHAF2, except in a small conserved domain present in all SDHAF2 sequences (Figure 2). Knockdown plants for this Arabidopsis SDHAF2 had decreased SDH enzymatic activity and a lowered amount of flavinated SDH1 in isolated mitochondria [5]. A putative Arabidopsis ortholog for human SDHAF1 exists, but also has low amino acid sequence similarity outside one key region of the protein (45\% identity in a $40 \mathrm{AA}$ window, Figure 2). This protein is predicted to be targeted to mitochondria but has not been experimentally identified in plant mitochondria to date [5]. Other plant genomes have orthologs of the SDHAF2 and the putative SDHAF1 of Arabidopsis. Another Arabidopsis protein, frataxin, has been experimentally implicated in assembly of Fe-S in SDH in plants $[15,16]$. However, there are three different Fe-S clusters in SDH2, and it is still not clear whether frataxin and SDHAF1 are responsible for independent insertion of different Fe-S clusters, or whether they work together in Fe-S insertion. To complicate matters further, frataxin's role is not specific to SDH, but has a broad role in assembly of Fe$S$ clusters for proteins in plant mitochondria $\left[15,166^{\circ}\right.$.

The four classical SDH1-4 subunits found in all eukaryotes form $\sim 110 \mathrm{kDa}$ native complexes. The plant SDH native complex appears to have a series of unknown function accessory subunits and a native mass of $\sim 160 \mathrm{kDa}$ (Figure 1). These subunits have been resolved by blue native (BN)-SDS-PAGE in a number of dicotyledonous plants and identified in Arabidopsis by peptide mass spectrometry $[17,18]$. Termed SDH5, 6, 7 and 8, these subunits have no known function and their sequences do not contain clear functional motifs, but together they may represent a secondary peripheral activity of the SDH complex in plants [18]. This could be analogous to the secondary functions already found for accessory and integral subunits of plant respiratory complex I and III $[19,20]$. The genome of rice contains 
clear orthologs of SDH5, SDH6 and SDH7, but to date no SDH8 ortholog has been defined in this any other plant species [1]. Interestingly, BN/SDS-PAGE separations of SDH from monocotyledonous plants, including rice and barley, typically observe a smaller SDH native complex than in Arabidopsis, potato and bean [1,17,21]. This may indicate a difference in the stability or composition of the larger plant SDH complex between plants. Recently, a native SDH complex in the monocot Symplocarpus renifolius was reported to be nearly $340 \mathrm{kDa}$ in size. It contained a range of smaller molecular mass subunits [22] and is the largest native SDH complex reported to our knowledge.

\section{The effect of SDH loss on plant phenotypes}

A series of mutants in different subunits have been recently reported that show the impact of lowering or removing SDH function from plant cells. These reports have shown that differential changes in organic acid levels, altered respiration rates in specific tissues and changes in mitochondrial ROS generation characteristics are likely to be the key reasons for this diversity of SDH dysfunction phenotypes.

The flavoprotein SDH1 is the site of succinate binding, is the basis for the allosteric activation of SDH by ATP and houses the first cofactor of SDH - a flavin covalently attached to a histidine residue. There are two genes for SDH1 in Arabidopsis, SDH11 (At5g66760) and SDH1-2 (At2g18450). Knockout of SDH1-2 did not affect the growth or development of Arabidopsis [23], SDH1-2 was expressed at a very low transcript level and has not been definitively identified at the protein level. In contrast, knockout of SDH1-1 led to failure of gameophyte development. Decrease of SDH1-1 by RNA interference resulted in pollen abortion and a reduced seed set [23]. Heterozygous $S D H 1-1 / s d h 1-1$ plants were recently reported to have low SDH activity but improved photosynthesis as well as improved growth in nitrogen-limiting conditions due to altered stomatal conductance and nitrogen assimilation, respectively (Figure 3, green boxes) [7]. The stomatal aperture and density in leaves were higher in both $S D H 1-1 / s d h 1-1$ plants and sdh1-1 RNAi plants [7]. Metabolite analysis revealed succinate was increased by 2-fold in these mutants, while some amino acids such as proline and glutamine were significantly lower in abundance [ $7^{\circ}$ ]. It has been speculated that metabolic alterations caused by less $\mathrm{SDH} 1$ could be the key basis for the significant changes in stomatal function, 
photosynthesis and nitrogen assimilation in these mutants [7"] (Figure 3). In contrast, knockdown of Arabidopsis SDHAF2 (At5g51040) which is the assembly factor for SDH1, showed a specific inhibition of root growth, but normal leaf growth (Figure 3, blue boxes) $\left[5^{\circ}\right]$. The low SDH activity in sdhaf2, did not affect photosynthetic rate or stomatal conductance $\left[5^{\circ}\right]$, which is very different from $\mathrm{SDH} 1$ deficient lines in Arabidopsis (Figure 3). The decrease of primary root elongation rate may be explained by the decreased rate of root tip respiration in these plants [5"]. Knockout of SDHAF2 in Arabidopsis lead to seed abortion, indicating its essential role early in seed development [5"] perhaps akin to the strong phenotype of SDH1-1 knockouts in Arabidopsis [23].

The iron-sulfur subunit (SDH2) of SDH in Arabidopsis is encoded by three genes, SDH2-1 (At3g27380), SDH2-2 (At5g40650) and SDH2-3 (At5g65165). SDH2-1 and SDH2-2 in Arabidopsis have distinct cell specific expression patterns and promoter activities, in fact only the $S D H 2-2$ isoform is expressed in root tips at a high level [24]. The knockout of $S D H 2-1$ did not have any phenotype, indicating functional redundancy between $S D H 2-1$ and $S D H 2-2$ [24]. $S D H 2-3$ is specifically expressed in the embryo during seed development [25] and the disruption of $S D H 2-3$ alone delayed seed germination [26]. In tomato plants, RNA interference of SDH2 increased the rate of net photosynthesis and growth (Figure 3, green boxes), mainly due to greater stomatal aperture in these lines [4"]. This observation is similar to that reported for the Arabidopsis heterozygous $S D H 1-1 /$ sdh1-1 plants mentioned above [7"]. The inhibition of the SDH2-2 expression using a guard cell-specific MYB60 promoter had no effect on changes in stomatal aperture or rates of photosynthesis in the mutant, suggesting an unexpected role for SDH in mesophyll cells that controlled stomatal function [4"]. A model has been proposed of SDH regulation of stomatal aperture by alteration of malate and fumarate levels [4"].

The direction and extent of changes in organic acid abundances in SDH1 and SDH2 plant mutants vary widely between reports and the tissues studied (Figure 3, white boxes). Also, SDH is more abundant in non-green tissues, such as roots, while other respiratory complexes such as complex I are more abundant in green tissues, such as leaves [27]. This could indicate a potential difference in the metabolic requirements in leaves and roots for these two major protein complexes of 
mitochondrial respiration. Complex I mutants are often small plants with equally reduced growth of both root and shoot [28-30], which clearly contrasts with the plant SDH mutants that have been reported with normal or enhanced leaf growth. In humans, the impact of organic acid changes following SDH inhibition has not been linked to the consequences of fumarate depletion, but to rather succinate accumulation and its impact as a competitive inhibitor of a-ketoglutarate-dependent enzymes [31]. These succinate-dependent inhibitions of other enzymes spread the impact of SDH inhibition from a respiration limitation to a wider impact on pseudohypoxia induction [32,33], sulphur metabolism and histone demethylation [31]. Details of how elevated succinate levels might inhibit plant cell functions are still unknown and require investigation.

Complexes I and III and the UQ cycle are generally considered as the main sites of reactive oxygen species (ROS) production in the eukaryotic respiratory chain [34], however, evidence is emerging that succinate-dependent $\mathrm{H}_{2} \mathrm{O}_{2}$ production by mitochondria is also very significant [35] and can be modulated independently of $\mathrm{H}_{2} \mathrm{O}_{2}$ production from other sites in the electron transport chain [36]. Structural analysis of SDH has proposed that SDH can produce ROS at the FAD- or UQbinding sites though single electron reductions of $\mathrm{O}_{2}$ (Figure 3) [37]. Recent in vitro experiments have proved this is true in both the forward and reverse reactions of the enzyme, i.e. as a succinate oxidase and as a fumarate reductase [38]. The mutation of SDH subunits that raise ROS formation from the complex have caused oxidative stress and ageing in nematodes [39] and caused cancers in humans [3]. In plants, a point mutation in $S D H 1-1$ (dsr1), at a conserved region in the substrate binding pocket, led to the significant reduction of SDH activity and paradoxically a significant lowering of mitochondrial ROS production (Figure 3, yellow boxes) [6"]. This mutation interrupted salicylic acid-mediated expression of specific stress responsive transcripts and defence genes in Arabidopsis (Figure 3), indicating the role of $\mathrm{SDH}$ derived ROS in plant defense gene expression. These mitochondrial ROS-deficient plants exhibited increased susceptibility to specific bacterial pathogen $(P$. syringae Pst DC3000) and fungal (A. brassicicola, R. solani) (Figure 3) as a result [6"]. Coupling these discoveries with the recent deployment of SDH inhibitors as pesticides against major fungal pathogens of crops $\left[40,41^{\circ}, 42\right]$ raised questions 
about whether inhibitor actions across plant and fungi SDH may contribute to the success of these pesticides.

Collectively these observations are allowing us to build an integrated working model of how SDH dysfunction impacts plant function, and ipso facto, the wider roles of SDH function in plant growth and development (Figure 3). Some aspects of the four main effects observed in plants (blue, yellow, green and white boxes) have clear mammalian counterparts (grey boxes), while others still remain as plant-specific consequences of altered succinate oxidation.

\section{Conclusions}

Loss or mutation of SDH subunits in plants can result in the decrease of SDH activity and respiration rate, accumulation of succinate, changes to the abundance of other organic acids, and variations in the production of mitochondrial ROS. As a research community, we still don't understand how tissue specific effects in SDH mutants may be linked to the clear tissue specific expression patterns of SDH isoforms in plants or how these change SDH kinetics or function. Furthermore, investigations on how different plant SDH mutants influence ROS production and downstream signals may provide new clues to explain the observed differences that mitochondrial ROS play in cellular REDOX signal transduction and also in plant mutant phenotypes. Future analysis of the functional importance of the variation of SDH3 and SDH4 in plants, combined with SDH UQ binding inhibitor studies, will enhance our understanding of how these subunits diverge and also define any side-effects on plants that may be caused by the use of SDH inhibitors as crop fungicides (Figure 1). Clearly it seems that SDH, once considered a simple and well known enzyme, plays a complicated role in plants that is only now beginning to be understood. 
Figure captions:

Figure 1. SDH composition, assembly factors and inhibitors. The plant mitochondrial succinate dehydrogenase (SDH, complex II), like its mammalian counterpart, is composed of four subunits: two hydrophilic matrix facing proteins (SDH1 and SDH2) and two largely hydrophobic proteins (SDH3 and SDH4) anchoring the complex in the membrane. SDH1 is a flavoprotein containing a covalently bound FAD, SDH2 is an iron-sulfur (Fe-S) protein which contains three Fe-S clusters. SDH3 and 4 are two integral membrane proteins that binding heme to form the b-type cytochrome. In addition, the plant mitochondrial SDH complex contains four plant-specific hydrophilic subunits (SDH5, 6, 7 and 8) with unknown functions. Two SDH assembly factors, SDHAF1 and SDHAF2, are required for insertion of Fe-S and FAD into SDH2 and SDH1, respectively. Frataxin has a role in Fe-S and/or heme synthesis for SDH. During the oxidation of succinate to form fumarate, electrons $\left(\mathrm{e}^{-}\right)$are passed through FAD, Fe-S centres and then reduce ubiquinone $(\mathrm{Q})$ to ubiquinol $\left(\mathrm{QH}_{2}\right)$. The succinate pocket is acted upon by substratelevel inhibitors (such as the succinate-analogues: malonate, malate, oxaloacetate (OAA) and also by ATP that activates the enzyme by inhibitor removal. A different class of inhibitors (SDHI) bind in the ubiquinone pocket (e.g. carboxin, which is used as a fungicide on crop plants).

Figure 2. Similarity of SDH subunits and assembly factors in selected eukaryotes. Protein sequences of SDH subunits and its assembly factors from the model plant Arabidopsis thaliana (A), the model crop plant Oryza sativa, rice (R), the model fungi Saccharomyces cerevisiae, yeast $(\mathbf{Y})$, and Human $(\mathbf{H})$ were compared with BLASTP. The recently sequenced fungus Mycosphaerella graminicola (M) was included because it is a major disease of crop plants and succinate dehydrogenase inhibitors (SDHI) are widely used to control its spread. The numbers in boxes represent alignment bit scores between paired protein sequences from BLAST analysis. Amino acid length of Arabidopsis proteins are given in the upper right side of individual panel. Rice Os08g17650 and Os11g32480 are potential candidates for SDHAF1, SDHAF2 in this species, and Arabidopsis At2g39725 is a potential candidate for SDHAF1 based on sequence similarity but all three require further experimental evidence to definitively prove their function. The $\mathrm{SDH} 1,2,3,4$, 
SDHAF1 and SDHAF2 accession numbers used for sequence alignment are: for Arabidopsis, At5g66760, At3g27380, At5g09600, At2g46505, At2g39725, At5g51040; for Rice, Os07g04240, Os08g02640, Os02g02940, Os01g70980, Os08g17650, Os11g32480; for Yeast, Q00711, P21801, P33421, P37298, Q3E785, Q08230; for Mycosphaerella graminicola, XP_003857174.1, XP_003850753.1, XP_003850451.1, XP_003853609.1, XP_003857429.1, XP_003857639.1; and for Human, P31040, P21912, Q6IAQ2, O14521, A6NFY7, NP_060311, respectively.

Figure 3. Mutations, effects and phenotypes of SDH loss in plants and animals. Dysfunction of SDH in plants, such as tomato sdh2 antisense lines [4"],

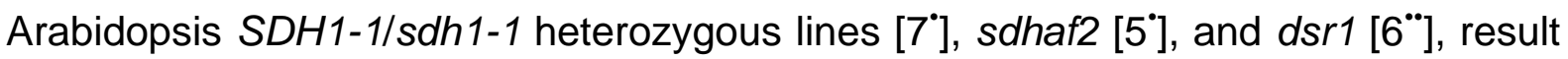
in the reduction of SDH activities and the accumulation of succinate. However, these lines do not show the same phenotypes. Tomato sdh2 antisense lines and Arabidopsis sdh1-1 heterozygotes have higher organic acid contents, increased stomatal conductance and higher rates of photosynthesis (Green box). A knockdown line of sdhaf2 had low root tip respiration and inhibited primary root elongation, without any impact on stomata or photosynthesis (Blue box). The dsr1 mutant (carrying a point mutation of SDH1-1) had reduced mitochondrial reactive oxygen species (ROS) production and affected expression of nuclear genes (such as GSTs and HSPs), making plants susceptible to bacterial and fungi diseases (Yellow box). In humans, dysfunction of SDH either causes neurological and blood disorders linked to lowered respiration rate or tumorigenesis linked to elevated mitochondrial reactive oxygen species (ROS) formation [2,3] (Grey box). The accumulation of succinate rather than changes in the abundance of fumarate and malate have been linked to organic acid based impact of SDH inhibition in humans. Elevated succinate inhibits a-ketoglutarate-dependent enzymes and propyl hydroxylases then cause downstream cellular toxicity [31,32] (Grey box). 
Acknowledgements:

The work of $\mathrm{SH}$ and $\mathrm{AHM}$ is funded by the Australian Research Council through the ARC Centre of Excellence in Plant Energy Biology (CE0561495). AHM was funded as an ARC Australian Future Fellow (FT110100242).

\section{References and recommending reading}

- of special interest

•• of outstanding interest

1. Huang S, Taylor NL, Narsai R, Eubel H, Whelan J, Millar AH: Functional and composition differences between mitochondrial complex II in Arabidopsis and rice are correlated with the complex genetic history of the enzyme. Plant Mol Biol 2010, 72:331-342.

2. Rutter J, Winge DR, Schiffman JD: Succinate dehydrogenase - Assembly, regulation and role in human disease. Mitochondrion 2010, 10:393-401.

3. Bardella C, Pollard PJ, Tomlinson I: SDH mutations in cancer. Biochim Biophys Acta 2011, 1807:1432-1443.

4. Araujo WL, Nunes-Nesi A, Osorio S, Usadel B, Fuentes D, Nagy R, Balbo I, Lehmann M, Studart-Witkowski C, Tohge T, et al.: Antisense Inhibition of the Iron-Sulphur Subunit of Succinate Dehydrogenase Enhances Photosynthesis and Growth in Tomato via an Organic Acid-Mediated Effect on Stomatal Aperture. Plant Cell 2011, 23:600-627.

..Using antisense to SDH2 in tomato, the authors show that inhibition of SDH increased carbon dioxide assimilation by up to $25 \%$ and mature plants has increased biomass. This phenomenon was linked to the rate of transpiration and stomatal conductance, charting a role of TCA cycle intermediates in photosynthetic metabolism the regulation of stomatal aperture.

5. Huang S, Taylor NL, Ströher E, Fenske R, Harvey Millar A: Succinate dehydrogenase assembly factor 2 is needed for assembly and activity of mitochondrial complex II and for normal root elongation in Arabidopsis. Plant J 2013, 73:429-441.

-Specific assembly factors for SDH were unknown in plants until the new defined homologs of mammalian assembly factors discovered in 2009 were investigated. These authors provide the first evidence that an SDHAF2 homolog is required for SDH1 flavination in plants and show that knockdown of an assembly factor encoded by one gene has different effects that knockout of specifc isoforms of SDH subunits, with the clearest effect on root tip growth.

6. Gleason C, Huang S, Thatcher LF, Foley RC, Anderson CR, Carroll AJ, Millar AH, Singh KB: Mitochondrial complex II has a key role in mitochondrialderived reactive oxygen species influence on plant stress gene regulation and defense. Proc Natl Acad Sci USA 2011, 108:10768-10773.

- The discovery of a mutant in a forward genetic screen for fungal defence in Arabidopsis by these authors, which turned out to be a site mutation in SDH1, raised an entirely new perspective on SDH function and the role of ROS signalling from plant mitochondria. Optimal function of SDH1 is required for salicylic acid mediated defence gene expression in Arabidopsis, and mutation makes plants more susceptible to both bacterial and fungal pathogens.

7. Fuentes D, Meneses M, Nunes-Nesi A, Araujo WL, Tapia R, Gomez I, Holuigue L, Gutierrez RA, Fernie AR, Jordana X: A Deficiency in the Flavoprotein of Arabidopsis Mitochondrial Complex II Results in Elevated 
Photosynthesis and Better Growth in Nitrogen-Limiting Conditions. Plant Physiol 2011, 157:1114-1127.

-Homozygous SDH1 mutants in Arabidopsis are lethal, so to biochemically study this lesion these authors used heterozygous $S D H 1-1 /$ sdh $1-1$ mutant plants displaying a $30 \%$ reduction in $\mathrm{SDH}$ activity. These plants displayed significantly higher $\mathrm{CO}_{2}$ assimilation rates and enhanced growth linked to stomatal aperture differences, and also altered amino acid metabolism that allowed plants to grow considerably better in nitrogen-limiting conditions.

8. Lemire BL, Oyedotun KS: The Saccharomyces cerevisiae mitochondrial succinate:ubiquinone oxidoreductase. Biochim Biophys Acta 2002, 1553:102-116.

9. Sun F, Huo X, Zhai Y, Wang A, Xu J, Su D, Bartlam M, Rao Z: Crystal structure of mitochondrial respiratory membrane protein Complex II. Cell 2005, 121:1043-1057.

10. Burger C, Lang F, Reith M, Gray W: Genes encoding the same three subunits of respiratory complex II are present in the mitochondrial DNA of two phylogenetically distinct eukarytoes. Proc Natl Acad Sci USA 1996, 93:2328-2332.

11. Adams KL, Rosenblueth M, Qiu Y-L, Palmer JD: Multiple losses and transfers to the nucleus of two mitochondrial succinate dehydrogenase genes during angiosperm evolution. Genetics 2001, 158:1289-1300.

12. Hao H-X, Khalimonchuk O, Schraders M, Dephoure N, Bayley J-P, Kunst H, Devilee P, Cremers CWRJ, Schiffman JD, Bentz BG, et al.: SDH5, a Gene Required for Flavination of Succinate Dehydrogenase, Is Mutated in Paraganglioma. Science 2009, 325:1139-1142.

13. Ghezzi D, Goffrini P, Uziel G, Horvath R, Klopstock T, Lochmuller H, D'Adamo P, Gasparini $P$, Strom TM, Prokisch $H$, et al.: SDHAF1, encoding a LYR complex-II specific assembly factor, is mutated in SDH-defective infantile leukoencephalopathy. Nat Genet 2009, 41:654-656.

14. Taylor NL, Heazlewood JL, Millar AH: The Arabidopsis thaliana 2-D gel mitochondrial proteome: Refining the value of reference maps for assessing protein abundance, contaminants and post-translational modifications. Proteomics 2011, 11:1720-1733.

15. Busi MV, Maliandi MV, Valdez H, Clemente M, Zabaleta EJ, Araya A, GomezCasati DF: Deficiency of Arabidopsis thaliana frataxin alters activity of mitochondrial Fe-S proteins and induces oxidative stress. Plant $J$ 2006, 48:873-882.

16. Maliandi MV, Busi MV, Turowski VR, Leaden L, Araya A, Gomez-Casati DF: The mitochondrial protein frataxin is essential for heme biosynthesis in plants. FEBS J 2011, 278:470-481.

-Frataxin's role in iron metabolism in plant mitochondria has been unclear until these authors found that frataxin deficiency in Arabidopsis decreased activity of both aconitase and succinate dehydrogenase, despite the increased expression of the respective genes, indicating an important role for Arabidopsis thaliana frataxin homolog in assembly of these Fe$S$ containing enzymes. Transcripts of the heme biosynthetic pathway decreased, as did total heme content, substantiating a role for mitochondrial frataxin in the biogenesis of $\mathrm{Fe}$-S groups and the biosynthesis of heme groups in plants.

17. Eubel $H$, Jänsch $L$, Braun $H-P$ : New insights into the respiratory chain of plant mitochondria. Supercomplexes and a unique composition of Complex II. Plant Physiol 2003, 133:274-286.

18. Millar AH, Eubel H, Jansch L, Kruft V, Heazlewood JL, Braun HP: Mitochondrial cytochrome c oxidase and succinate dehydrogenase complexes contain plant specific subunits. Plant Mol Biol 2004, 56:77-90. 
19. Braun HP, Schmitz UK: The bifunctional cytochrome c reductase/processing peptidase complex from plant mitochondria. J Bioenerg Biomembr 1995, 27:423-436.

20. Klodmann J, Sunderhaus S, Nimtz M, Jansch L, Braun HP: Internal architecture of mitochondrial complex I from Arabidopsis thaliana. Plant Cell 2010, 22:797-810.

21. Heazlewood JL, Tonti-Filippini JS, Gout AM, Day D, Whelan J, Millar AH: Experimental analysis of the arabidopsis mitochondrial proteome highlights signaling and regulatory components, provides assessment of targeting prediction programs, and Indicates plant-specific mitochondrial proteins. Plant Cell 2004, 16:241-256.

22. Kakizaki $\mathrm{Y}$, Moore AL, Ito $\mathrm{K}$ : Different molecular bases underlie the mitochondrial respiratory activity in the homoeothermic spadices of Symplocarpus renifolius and the transiently thermogenic appendices of Arum maculatum. Biochem J 2012, 445:237-246.

23. León $G$, Holuigue L, Jordana X: Mitochondrial Complex II Is Essential for Gametophyte Development in Arabidopsis. Plant Physiol 2007, 143:15341546.

24. Elorza A, Leon G, Gomez I, Mouras A, Holuigue L, Araya A, Jordana X: Nuclear SDH2-1 and SDH2-2 Genes, Encoding the Iron-Sulfur Subunit of Mitochondrial Complex II in Arabidopsis, Have Distinct Cell-Specific Expression Patterns and Promoter Activities. Plant Physiol 2004, 136:4072-4087.

25. Elorza A, Roschzttardtz H, Gomez I, Mouras A, Holuigue L, Araya A, Jordana X: A nuclear gene for the iron-sulfur subunit of mitochondrial complex II is specially expressed during Arabidopsis seed development and germination. Plant Cell Physiol 2006, 47:14-21.

26. Roschzttardtz H, Fuentes I, Vasquez M, Corvalan C, Leon G, Gomez I, Araya A, Holuigue L, Vicente-Carbajosa J, Jordana X: A Nuclear Gene Encoding the Iron-Sulfur Subunit of Mitochondrial Complex II Is Regulated by B3 Domain Transcription Factors during Seed Development in Arabidopsis. Plant Physiol 2009, 150:84-95.

27. Peters K, Nießen M, Peterhänsel C, Späth B, Hölzle A, Binder S, Marchfelder A, Braun H-P: Complex I-complex II ratio strongly differs in various organs of Arabidopsis thaliana. Plant Mol Biol 2012, 79:273-284.

28. Koprivova A, des Francs-Small CC, Calder G, Mugford ST, Tanz S, Lee B-R, Zechmann B, Small I, Kopriva S: Identification of a Pentatricopeptide Repeat Protein Implicated in Splicing of Intron 1 of Mitochondrial nad7 Transcripts. J Biol Chem 2010, 285:32192-32199.

29. Meyer EH, Tomaz T, Carroll AJ, Estavillo G, Delannoy E, Tanz SK, Small ID, Pogson BJ, Millar AH: Remodeled Respiration in ndufs4 with Low Phosphorylation Efficiency Suppresses Arabidopsis Germination and Growth and Alters Control of Metabolism at Night. Plant Physiol 2009, 151:603-619.

30. Kuhn K, Richter U, Meyer EH, Delannoy E, Falcon de Longevialle A, O'Toole N, Borner T, Millar AH, Small ID, Whelan J: Phage-Type RNA Polymerase RPOTmp Performs Gene-Specific Transcription in Mitochondria of Arabidopsis thaliana. Plant Cell 2009, 21:2762-2779. 
31. Smith $E H$, Janknecht $R$, Maher LJ: Succinate inhibition of $\alpha$-ketoglutaratedependent enzymes in a yeast model of paraganglioma. Human Mol Gen 2007, 16:3136-3148.

32. King A, Selak MA, Gottlieb E: Succinate dehydrogenase and fumarate hydratase: linking mitochondrial dysfunction and cancer. Oncogene 2006, 25:4675-4682.

33. Mackenzie ED, Selak MA, Tennant DA, Payne LJ, Crosby S, Frederiksen CM, Watson DG, Gottlieb E: Cell-Permeating a-Ketoglutarate Derivatives Alleviate Pseudohypoxia in Succinate Dehydrogenase-Deficient Cells. Mol Cell Biol 2007, 27:3282-3289.

34. Murphy MP: How mitochondria produce reactive oxygen species. Biochem $J$ 2009, 417:1-13.

35. Tahara E, Navarete F, Kowaltowski A: Tissue-, substrate-, and site-specific characteristics of mitochondrial reactive oxygen species generation. Free Radic Biol Med 2009, 46:1283-1297.

36. Venditti $P$, Di-Stefano L, Di-Meo S: Vitamin E reduces cold-induced oxidative stress in rat skeletal muscle decreasing mitochondrial $\mathrm{H}(2) \mathrm{O}(2)$ release and tissue susceptibility to oxidants. Redox Rep 2009, 14:167-175.

37. Yankovskaya V, Horsefield R, Törnroth S, Luna-Chavez C, Miyoshi H, Léger C, Byrne B, Cecchini G, Iwata S: Architecture of Succinate Dehydrogenase and Reactive Oxygen Species Generation. Science 2003, 299:700-704.

38. Quinlan CL, Orr AL, Perevoshchikova IV, Treberg JR, Ackrell BA, Brand MD: Mitochondrial Complex II Can Generate Reactive Oxygen Species at High Rates in Both the Forward and Reverse Reactions. $J$ Biol Chem 2012, 287:27255-27264.

39. Ishii N, Fujii M, Hartman P, Tsuda M, Yasuda K, Senoo-Matsuda N, Yanase S, Ayusawa $D$, Suzuki $K$ : A mutation in succinate dehydrogenase cytochrome b causes oxidative stress and ageing in nematodes. Nature 1998, 394:694-697.

40. Scalliet G, Bowler J, Luksch T, Kirchhofer-Allan L, Steinhauer D, Ward K, Niklaus $M$, Verras $A$, Csukai $M$, Daina $A$, et al.: Mutagenesis and Functional Studies with Succinate Dehydrogenase Inhibitors in the Wheat Pathogen Mycosphaerella graminicola. PLOS ONE 2012, 7:e35429.

41. Fraaije BA, Bayon C, Atkins S, Cools HJ, Lucas JA, Fraaije MW: Risk assessment studies on succinate dehydrogenase inhibitors, the new weapons in the battle to control Septoria leaf blotch in wheat. Mol Plant Path 2012, 13:263-275.

-Recent deployment of SDH inhibitors as a new line of defence against plant fungal pathogens is exemplified by the chemical control of Septoria leaf blotch, caused by Mycosphaerella graminicola. Because of their single-site specificity, these fungicides may be prone to resistance development and these authors developed and explored a library of carboxin-resistant mutants to define the $\mathrm{SDH} 2,3$ and 4 sites conferring resistance. Such studies will aid the implementation of anti-resistance strategies to prolong the costeffectiveness and lifetime of SDHI fungicides.

42. Veloukas T, Karaoglanidis GS: Biological activity of the succinate dehydrogenase inhibitor fluopyram against Botrytis cinerea and fungal baseline sensitivity. Pest Manag Sci 2012, 68:858-864. 
Inter-membrane space

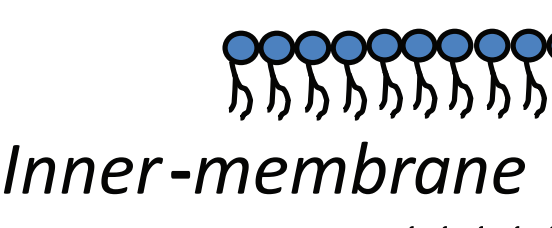

Matrix 9000000000

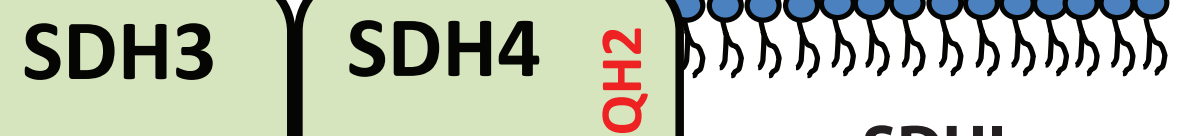
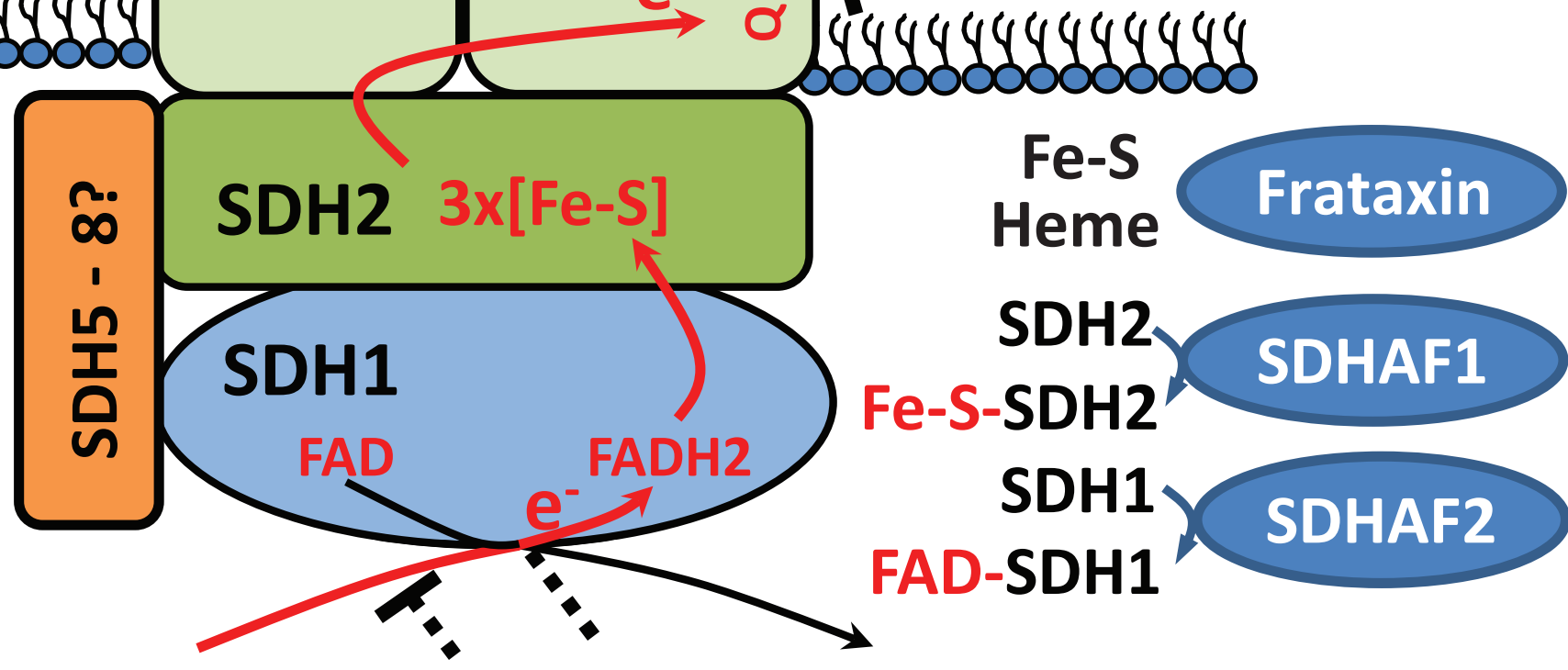

Succinate

OAA ATP malonate

Fumarate 

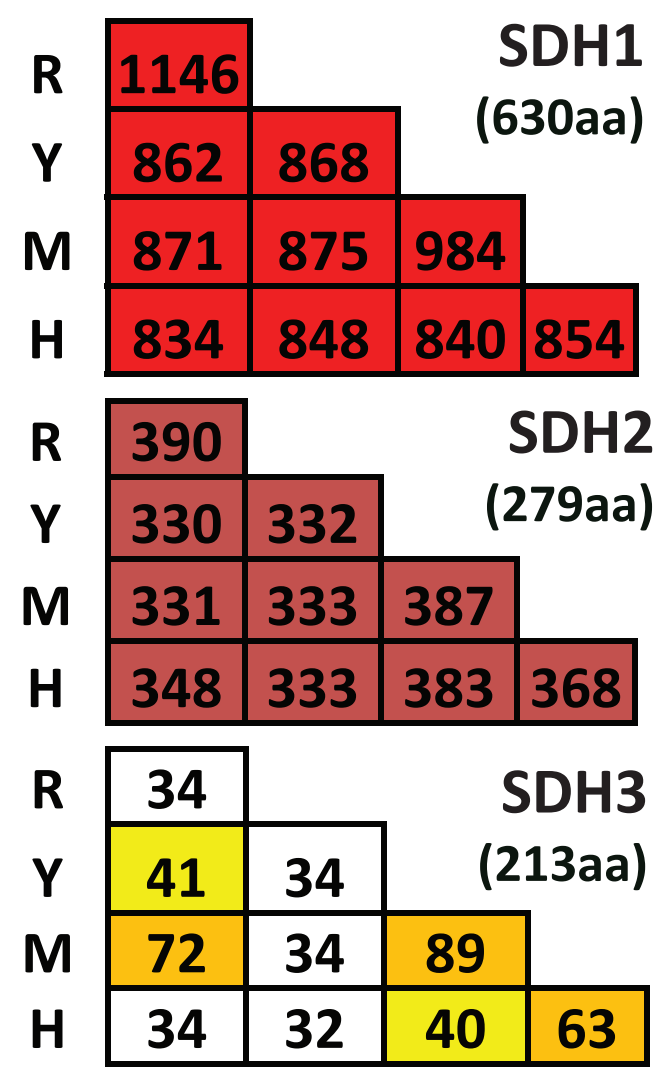

\begin{tabular}{|c|c|c|c|c|}
\hline $\mathbf{R}$ & 37 & & \multirow{2}{*}{\multicolumn{2}{|c|}{$\begin{array}{r}\text { SDH4 } \\
\text { (151aa) }\end{array}$}} \\
\hline$Y$ & 14 & 19 & & \\
\hline & 19 & 29 & 74 & \\
\hline & 37 & 20 & - & 37 \\
\hline
\end{tabular}

\begin{tabular}{|c|c|c|c|c|}
\hline $\mathbf{R}$ & 111 & & \multirow{2}{*}{\multicolumn{2}{|c|}{$\begin{array}{r}\text { SDHAF1 } \\
(90 a a)\end{array}$}} \\
\hline $\mathbf{Y}$ & 56 & 59 & & \\
\hline & 59 & 62 & 70 & \\
\hline & 49 & 54 & 40 & 50 \\
\hline
\end{tabular}
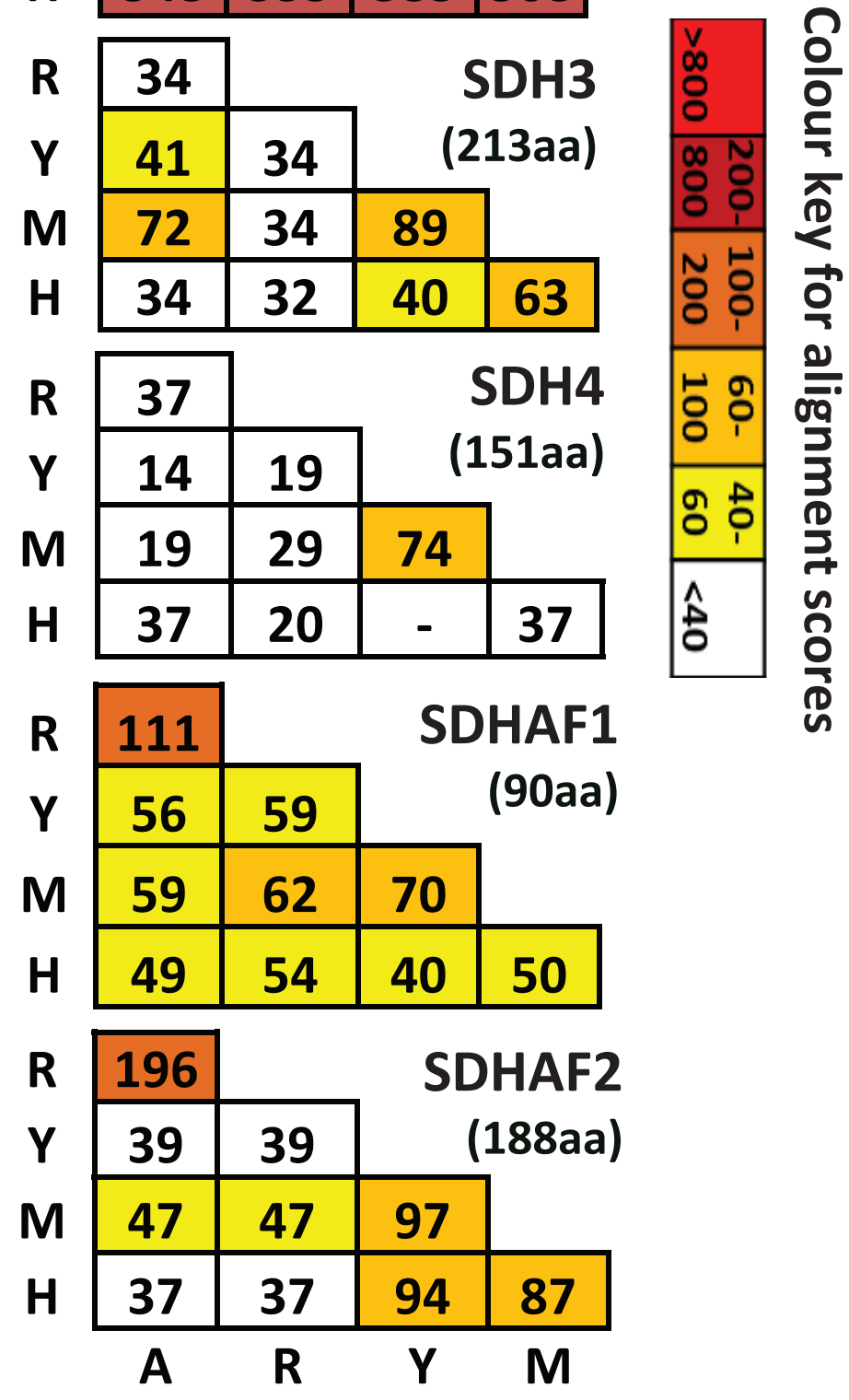
